\title{
Desempenho agronômico e variabilidade genética em genótipos de couve
}

\author{
Alcinei Mistico Azevedo(1), Valter Carvalho de Andrade Júnior ${ }^{(2)}$, Carlos Enrrik Pedrosa ${ }^{(2)}$, \\ José Sebastião Cunha Fernandes ${ }^{(2)}$, Nermy Ribeiro Valadares ${ }^{(2)}$, \\ Marcos Aurélio Miranda Ferreira ${ }^{(2)}$ e Rafael Augusto do Valle Martins ${ }^{(2)}$
}

\begin{abstract}
(1)Universidade Federal de Viçosa, Avenida PH Rolfs, s/no, CEP 36570-000 Viçosa, MG. E-mail: alcineimistico@hotmail.com (2)Universidade Federal dos Vales do Jequitinhonha e Mucuri, Campus JK, Alto da Jacuba, Rodovia MGT-367, Km 583, no 5.000, CEP 39100-000 Diamantina, MG. E-mail: valterjr@ufvjm.edu.br, carlosenrrik@yahoo.com.br, cunha.fernandes@ufvjm.edu.br, nermyvaladares@hotmail.com, marcaumife2010@hotmail.com,rafa_montesclaros@yahoo.com.br
\end{abstract}

Resumo - O objetivo deste trabalho foi avaliar o desempenho agronômico de acessos de couve e estimar os parâmetros genéticos e a correlação entre características de interesse para o melhoramento. $\mathrm{O}$ experimento foi realizado em delineamento de blocos ao acaso, com 30 genótipos de couve, entre os quais, três cultivares comerciais, de diferentes empresas. Foram utilizadas quatro repetições, com cinco indivíduos por parcela. Verificou-se variabilidade genética entre os genótipos, com predominância dos efeitos genéticos sobre os ambientais, o que indica a possibilidade de se obterem ganhos genéticos representativos com o melhoramento. As características importantes para o melhoramento da espécie foram: comprimento e largura de folha, diâmetro de pecíolo, área foliar, altura de planta, número de brotações e massa de folhas secas. Os genótipos comerciais apresentaram menor área foliar, massa de matéria seca de folhas, altura de planta, comprimento e largura de folha, comprimento de pecíolo, e número de brotações e de folhas comerciais.

Termos para indexação: Brassica oleracea var. acephala, correlação fenotípica, correlação genotípica, herdabilidade, parâmetros genéticos.

\section{Agronomic performance and genetic variability in kale genotypes}

\begin{abstract}
The objective of this work was to evaluate the agronomic performance of kale genotypes and to estimate the genetic parameters and the correlation between traits of interest for breeding. The experiment was carried out in a randomized block design, with 30 kale genotypes, with three commercial cultivars, from three different companies. It was used four replicates, with five individuals per plot. Variability was significant among genotypes, with predominance of genetic effects over environmental ones, indicating the possibility of obtaining expressive genetic gains in breeding. The most important traits for breeding purposes were: leaf length and width, stem diameter, leaf area, plant height, number of shoots, and leaf dry weight. Commercial genotypes showed smaller leaf area, dry leaf mass, plant height, leaf length and width, petiole length, and number of sprouts and commercial leaves.
\end{abstract}

Index terms: Brassica oleracea var. acephala, phenotypic correlation, genotypic correlation, heritability, genetic parameters.

\section{Introdução}

A couve (Brassica oleracea var. acephala DC.) é uma hortaliça arbustiva anual ou bienal, cujo consumo no Brasil tem aumentado gradativamente (Novo et al., 2010b). Além de apresentar potencial anticarcinogênico, pois suas folhas são ricas em glucosinolatos, a couve possui elevado teor de flavonoides, vitaminas e nutrientes minerais (Moreno et al., 2006).

A espécie $B$. oleracea $(2 \mathrm{n}=18)$ apresenta autoincompatibilidade do tipo esporofítica, o que contribui para o aumento na variabilidade genética. Há várias pesquisas, em outros países, acerca da variabilidade genética em couve, avaliada por caracteres morfoagronômicos (Cartea et al., 2002; Nath et al., 2003; Balkaya \& Yanmaz, 2005; Vaishnava et al., 2006; Khan et al., 2009; Khatun et al., 2010; Belete et al., 2011; Yousuf et al., 2011) e moleculares (Okumus \& Balkaya, 2007; Koutita et al., 2008; Sjödin et al., 2008; Christensen et al., 2011). Entretanto, no Brasil, pesquisas nesta área são pouco frequentes, entre as quais destacam-se as de Sawazaki et al. (1997), Novo et al. (2010b) e Novo et al. (2010a), que caracterizaram genótipos de couve com descritores agromorfológicos, isoenzimáticos e RAPD. Portanto, são desejáveis mais pesquisas de caracterização, com vistas à seleção de genótipos superiores e de genitores para programas de melhoramento. 
Atualmente, o melhoramento genético de couve tem procurado selecionar genótipos com menor altura, menor número de brotações e maior número de folhas, para facilitar os tratos culturais e aumentar o rendimento por área.

Além da avaliação do desempenho agronômico de possíveis genitores, alguns estudos preliminares devem ser feitos para auxiliar na escolha de estratégias de melhoramento mais adequadas para a cultura e que permitam fazer inferências sobre a predição de ganhos com a seleção (Cruz et al., 2004). Entre esses estudos, destacam-se as estimativas de parâmetros genéticos como: variância genotípica, coeficiente de variação genética, herdabilidade e índice de variação, que refletem a proporção da variação fenotípica determinada pela variação genética ou genotípica para determinado caráter. Além disso, é importante que se determinem as correlações entre caracteres de interesse para o melhoramento, causadas por pleiotropia ou ligação entre os genes.

O objetivo deste trabalho foi avaliar o desempenho agronômico de acessos de couve do banco de germoplasma da Universidade Federal dos Vales do Jequitinhonha e Mucuri (UFVJM), e estimar os parâmetros genéticos e a correlação entre características de interesse para o melhoramento.

\section{Material e Métodos}

O experimento foi realizado no Setor de Olericultura, no campus JK da UFVJM, Diamantina, MG (18 $12^{\prime} 01^{\prime \prime S}, 43^{\circ} 34^{\prime} 20^{\prime \prime} \mathrm{W}$, altitude de $\left.1.387 \mathrm{~m}\right)$. No período de condução do experimento, a temperatura média foi de $18,3^{\circ} \mathrm{C}$, e a umidade relativa do ar de $80,3 \%$, com pequenas oscilações.

Foram avaliadas três cultivares comerciais e vinte e sete acessos de couve do banco de germoplasma da UFVJM, em delineamento de blocos ao acaso, com quatro repetições e cinco plantas por parcela. Dos acessos, 19 vieram de coletas em propriedades rurais na região de Diamantina, MG (UFVJM-2, UFVJM-3, UFVJM-4, UFVJM-5, UFVJM-7, UFVJM-8, UFVJM-9, UFVJM-10, UFVJM-13, UFVJM-19, UFVJM-21, UFVJM-22, UFVJM-24, UFVJM-26, UFVJM-27, UFVJM-30, UFVJM-32, UFVJM-34 e UFVJM-36), e oito acessos foram doados pela Universidade Federal de Lavras (UFLA-1, UFLA-3, UFLA-6, UFLA-8, UFLA-5, UFLA-10,
UFLA-11 e UFLA-12). As cultivares comerciais avaliadas foram: couve-manteiga 900 legítima pé alto (Feltrin Sementes, Farroupilha, RS, Brasil); couve-manteiga Baby (Vidasul Sementes Ltda., Xanxerê, SC, Brasil); e couve-de-folha Manteiga Geórgia (Horticeres Sementes Ltda., Indaiatuba, SP, Brasil).

O experimento foi instalado em 28/07/2011. Para a formação das mudas pelo sistema de estaquia verde, coletaram-se brotações laterais do terço basal das plantas matrizes, com três a quatro centímetros de altura e dois folíolos. Os brotos foram plantados em bandejas de isopor de 72 células, preenchidas com substrato comercial Plantmax e mantidas em casa de vegetação por 30 dias, para garantir o melhor enraizamento das brotações. As mudas foram transplantadas para vasos com capacidade de 7,5 kg, preenchidos com Latossolo Vermelho distrófico e esterco bovino à proporção de 3:1. Para garantir o melhor pegamento das mudas, os vasos foram mantidos em casa de vegetação por 30 dias. Em 28/09/2011, os vasos foram levados para o campo, onde permaneceram até a coleta dos dados para a avaliação agronômica. As adubações foram realizadas de acordo com as recomendações para a cultura (Filgueira, 2008), e o controle de pulgões e lagartas foi feito manualmente, em dias alternados. Semanalmente, foram retiradas as folhas senescentes e as brotações, para possibilitar o melhor desenvolvimento das plantas.

No dia 2/1/2012 foram avaliados: altura das plantas (cm) a partir do nível do solo até a extremidade das folhas mais altas; diâmetro do caule $(\mathrm{mm})$, medido com paquímetro na metade da altura da planta; o comprimento e a largura do limbo foliar $(\mathrm{cm})$, tendo-se medido a $5^{\mathrm{a}}$ folha expandida mais nova; o número de folhas apropriadas para a comercialização, obtido pela contagem das folhas maiores que $15 \mathrm{~cm}$ e sem sinais de senescência; o número total de folhas na planta, obtido pela contagem de todas as folhas, tendo-se desconsiderado as não expandidas e as folhas existentes nas brotações; e o número de brotações.

$\mathrm{Na} 5^{\text {a }}$ folha expandida mais nova de cada planta, foram avaliados: diâmetro do centro do pecíolo ( $\mathrm{mm}$ ), medido com paquímetro na metade da extensão do pecíolo; comprimento do pecíolo $(\mathrm{cm})$ a partir da inserção no caule até o início do limbo foliar; a área foliar $\left(\mathrm{cm}^{2}\right)$, por meio de Scanner HP Photosmart C4480, com o auxílio do programa Image Pro Plus 4.5; 
e a massa de matéria seca, obtida por parcela, com secagem das folhas em estufa a $65^{\circ} \mathrm{C}$, até massa constante.

Para as variáveis cujos dados foram coletados em cada indivíduo, o modelo utilizado para a análise de variância foi $Y_{i j k}=m+t_{i}+b_{j}+e_{i j}+d_{i j k}$. Para a massa de matéria seca das folhas, cujos dados foram obtidos para as parcelas como um todo, utilizou-se o modelo $Y_{i j}=m+t_{i}+b_{j}+e_{i j}$, em que: $Y_{i j k}$ é a observação referente ao k-ésimo indivíduo, do j-ésimo bloco, do i-ésimo genótipo; $\mathrm{Y}_{\mathrm{ij}}$ é a observação referente ao j-ésimo bloco, do i-ésimo genótipo; $\mathrm{m}$ é a média geral da variável em análise; $t_{i}$ é o efeito do i-ésimo genótipo $(i=1,2, \ldots, I)$; $b_{\mathrm{j}}$ é o efeito do j-ésimo bloco $(\mathrm{j}=1,2, \ldots, \mathrm{J})$; $\mathrm{e}_{\mathrm{ij}}$ é o efeito da interação entre o i-ésimo genótipo do j-ésimo bloco ou efeito ambiental da ij-ésima parcela; $\mathrm{d}_{\mathrm{ijk}}$ é o efeito do k-ésimo indivíduo dentro da ij-ésima parcela. Todos os efeitos do modelo foram assumidos como aleatórios, em que: $\mathrm{K}$ é o número de plantas por parcela; $\mathrm{J}$ é o número de blocos; I é o número de genótipos; e $\overline{\mathrm{K}}$ é a média harmônica do número de plantas por parcela, já que houve número desigual de genótipos sobreviventes por parcela. Para a análise de variância, seguiu-se o modelo balanceado, conforme Cruz et al. (2004).

De acordo com as expressões estatísticas expostas por Cruz et al. (2004), foram calculadas as estimativas do coeficiente de variação ambiental dentro $\left(\mathrm{CVe}_{\text {dentro }}\right)$ e entre $\left(\mathrm{CVe}_{\text {entre }}\right)$ os tratamentos, do coeficiente de variação genética $(\mathrm{CVg})$, das variâncias genéticas $\left(\hat{\sigma}_{\mathrm{g}}^{2}\right)$, variância residual $\left(\hat{\sigma}_{\mathrm{e}}^{2}\right)$, variância dentro dos tratamentos $\left(\hat{\sigma}_{\mathrm{d}}^{2}\right)$, herdabilidade da média $\left(\mathrm{h}_{(\mathrm{m})}^{2}\right)$, herdabilidade do indivíduo no bloco $\left(\mathrm{h}_{(\mathrm{i}) \mathrm{bl}}^{2}\right)$, herdabilidade do indivíduo no experimento $\left(\mathrm{h}_{(\mathrm{i}) \exp }^{2}\right)$, herdabilidade da parcela no bloco $\left(\mathrm{h}_{(\mathrm{p}) \mathrm{bl}}^{2}\right)$, herdabilidade da parcela no experimento $\left(\mathrm{h}_{(\mathrm{i}) \exp }^{2}\right)$, índice de variação $\left(\mathrm{I}_{\mathrm{v}}\right)$ e ganho com a seleção (GS), tendo-se considerado a escolha dos $20 \%$ melhores.

Foi feita a verificação da hipótese de nulidade, pela estatística t, para os coeficientes de correlações fenotípicas e genotípicas.

Para atender às pressuposições da análise de variância, os dados obtidos por contagem foram submetidos à transformação $(\mathrm{x}+1)^{0,5}$ - para a melhor visualização, foram apresentados os valores originais nas tabelas. Procedeu-se à análise de variância e, quando identificada diferença significativa pelo teste $\mathrm{F}$, as médias dos tratamentos foram comparadas pelo teste de Scott-Knott, a 5\% de probabilidade.

\section{Resultados e Discussão}

As estimativas de $\mathrm{F}$ foram significativas para todas as características avaliadas (Tabela 1), o que indica a presença de variabilidade genética entre as amostras estudadas, o que também foi verificado por Cartea et al. (2002) e Novo et al. (2010a, 2010b). Os coeficientes de variação ambiental, entre e dentro dos tratamentos, foram maiores para área foliar, e menores para o número de folhas totais, o que indica influência maior do ambiente sobre a primeira característica e menor sobre a segunda. Em geral, a precisão experimental foi satisfatória, considerando-se os valores do coeficiente de variação experimental e a significância do efeito dos genótipos.

A existência de variabilidade genética entre as amostras foi confirmada pelos coeficientes de variação genética, que evidenciaram que a largura da folha e o número total de folhas apresentam menor variação genética. Segundo Freitas et al. (2007), maiores valores coeficientes de variação genética indicam a maior possibilidade de progressos genéticos com a seleção.

Verificaram-se valores de variância genética superiores aos de variância residual, o que mostra a predominância do efeito genético sobre o ambiental para todas as características, e indica condições favoráveis para o melhoramento. Para a variância dentro dos genótipos, resultante apenas do efeito do ambiente, encontraram-se valores relativamente altos.

As estimativas de herdabilidade foram menores para os caracteres largura da folha, número de folhas comerciais e área foliar, que são características mais importantes no melhoramento da couve. Esse resultado indica, de acordo com Sato et al. (2007), menor controle genético destes caracteres e menor possibilidade de se obterem progressos genéticos por meio da seleção, na comparação com os demais caracteres, especialmente com o comprimento do pecíolo, altura de planta e diâmetro do caule, que apresentaram maior herdabilidade.

$\mathrm{Na}$ avaliação da razão entre o coeficiente de variação genotípico e experimental $\left(\mathrm{I}_{\mathrm{v}}\right)$, observaram-se valores próximos ou maiores do que a unidade, para comprimento de pecíolo, altura de planta, diâmetro de caule, número de folhas totais e massa de matéria seca de folhas. Isto indica que, para estas características, há maior possibilidade de se obterem ganhos genéticos com a seleção, o que também se pôde verificar pelas estimativas de ganho de seleção (10,40 a 26,21\%), 
que tiveram altos valores para os melhores genótipos $(20 \%)$.

Das características relacionadas direta ou indiretamente com as dimensões da folha (comprimento de folha, largura de folha, comprimento de pecíolo, diâmetro de pecíolo, área foliar e massa de matéria seca de folhas), apenas o comprimento de pecíolo não apresentou correlação fenotípica ou genotípica com o comprimento e largura da folha (Tabela 2). A correlação da altura de planta com o comprimento e o diâmetro do pecíolo não foi significativa, e foi positiva e significativa com o comprimento de folha, largura de folha, área foliar, número de folhas comerciais e número de brotações. Contudo, a altura de planta apresentou correlação negativa com o diâmetro de caule e número total de folhas. Esta informação é condizente com as relatadas sobre cultivares comerciais que, além de apresentarem menor porte (Novo et al., 2010b), tendem a produzir menor quantidade de brotações e folhas menores, em comparação às variedades tradicionais.

Tabela 1. Estimativas do quadrado médio do genótipo $\left(\mathrm{QM}_{\mathrm{gen}}\right)$ e dos coeficientes de variação ambiental dentro $\left(\mathrm{CVe}_{\text {dentro }}\right)$ e entre $\left(\mathrm{CVe}_{\text {entre }}\right)$ genótipos, coeficiente de variação genética $\left(\mathrm{CV}_{\mathrm{g}}\right)$, das variâncias genéticas $\left(\sigma_{\mathrm{g}}^{2}\right)$, variância residual $\left(\sigma_{\mathrm{e}}^{2}\right)$, variâncias dentro $\left(\sigma_{\mathrm{d}}^{2}\right)$ de genótipos, herdabilidade média $\left(\mathrm{h}_{(\mathrm{m})}^{2}\right)$, herdabilidade do indivíduo no bloco $\left(\mathrm{h}_{(\mathrm{i}) \mathrm{bl}}^{2}\right)$, herdabilidade do indivíduo no experimento $\left(\mathrm{h}_{(\mathrm{i}) \exp }^{2}\right)$, herdabilidade da parcela no bloco $\left(\mathrm{h}_{(\mathrm{p}) \mathrm{bl}}^{2}\right)$, herdabilidade da parcela no experimento $\left(\mathrm{h}_{(\mathrm{i}) \exp }^{2}\right)$, índice de variação $\left(I_{v}\right)$ e ganho com a seleção (GS), em genótipos de couve.

\begin{tabular}{|c|c|c|c|c|c|c|c|c|c|c|c|}
\hline Parâmetro & $\mathrm{CF}$ & LF & $\mathrm{CP}$ & DP & $\mathrm{AF}$ & $\mathrm{AP}$ & $\mathrm{DC}$ & NFC & NTF & NB & MSF \\
\hline QMgen & $3.993 * *$ & $1,593^{* *}$ & $2,186 * *$ & $602 * *$ & $1.038 .524 * *$ & $75.412 * *$ & $3.378 * *$ & $28,71 * *$ & $48,02 * *$ & $69,45^{* *}$ & $2,11 * *$ \\
\hline $\mathrm{CVe}_{\text {dentro }}(\%)$ & 22,70 & 17,24 & 25,00 & 18,02 & 27,20 & 13,94 & 19,25 & 24,38 & 10,25 & 24,08 & - \\
\hline $\mathrm{CVe}_{\text {entre }}(\%)$ & 8,13 & 9,36 & 8,12 & 8,79 & 15,25 & 8,67 & 7,49 & 10,77 & 2,32 & 7,45 & 17,73 \\
\hline CVg (\%) & 13,83 & 10,22 & 27,18 & 13,71 & 18,82 & 17,18 & 18,69 & 14,22 & 10,20 & 16,87 & 23,42 \\
\hline$\sigma_{\mathrm{g}}^{2}$ & 5,89 & 2,13 & 3,72 & 0,92 & 1480,42 & 119,44 & 5,37 & 0,06 & 0,13 & 0,16 & 0,46 \\
\hline$\sigma_{\mathrm{e}}^{2}$ & 2,04 & 1,79 & 0,33 & 0,38 & 971,78 & 30,44 & 0,86 & 0,03 & 0,01 & 0,03 & 0,26 \\
\hline$\sigma_{\mathrm{d}}^{2}$ & 15,88 & 6,06 & 3,14 & 1,58 & 3093,31 & 78,71 & 5,70 & 0,17 & 0,13 & 0,33 & - \\
\hline $\mathrm{h}_{(\mathrm{m})}^{2}(\%)$ & 81,42 & 73,53 & 93,74 & 83,76 & 78,48 & 91,16 & 91,44 & 72,03 & 91,00 & 82,06 & 87,47 \\
\hline$h_{(i) b l}^{2}(\%)$ & 24,75 & 21,33 & 51,67 & 31,85 & 26,70 & 52,25 & 45,00 & 22,16 & 48,51 & 30,92 & - \\
\hline $\mathrm{h}_{(\mathrm{i}) \exp }^{2}(\%)$ & 24,70 & 19,82 & 50,01 & 32,03 & 25,36 & 48,80 & 45,01 & 20,90 & 47,93 & 30,54 & - \\
\hline $\mathrm{h}_{(\mathrm{p}) \mathrm{bl}}^{2}(\%)$ & - & - & - & - & - & - & - & - & - & - & 63,58 \\
\hline $\mathrm{h}_{(\mathrm{i}) \exp }^{2}(\%)$ & - & - & - & - & - & - & - & - & - & - & 62,01 \\
\hline$I_{v}$ & 0,61 & 0,59 & 1,09 & 0,76 & 0,69 & 1,23 & 0,97 & 0,58 & 1,00 & 0,70 & 1,32 \\
\hline GS (\%) & 19,20 & 10,40 & 24,90 & 13,57 & 21,52 & 15,73 & 26,21 & 17,19 & 15,36 & 24,18 & 25,97 \\
\hline
\end{tabular}

$\mathrm{CF}$, comprimento de folha; LF, largura de folha; CP, comprimento de pecíolo; DP, diâmetro do centro do pecíolo; AF, área foliar; AP, altura de planta; DC, diâmetro de caule; NFC, número de folhas comerciais; NTF, número total de folhas; e NB, número de brotações; MSF, massa de matéria seca de folhas. **Significativo a $1 \%$ pelo teste $\mathrm{F}$.

Tabela 2. Coeficientes de correlação fenotípica (acima da diagonal) e correlação genotípica (abaixo da diagonal), entre características avaliadas em 30 genótipos de couve.

\begin{tabular}{|c|c|c|c|c|c|c|c|c|c|c|c|}
\hline Caracteres & $\mathrm{CF}$ & LF & $\mathrm{CP}$ & DP & $\mathrm{AF}$ & AP & $\mathrm{DC}$ & NFC & NTF & NB & MSF \\
\hline$\overline{\mathrm{CF}}$ & - & $0,42 *$ & $0,15^{\text {ns }}$ & $0,69 * *$ & $0,74 * *$ & $0,46^{*}$ & $0,13^{\text {ns }}$ & $0,68 * *$ & $-0,56^{* *}$ & $0,16^{\mathrm{ns}}$ & $0,55^{* *}$ \\
\hline $\mathrm{LF}$ & $0,42 *$ & - & $0,25^{\text {ns }}$ & $0,42 *$ & $0,82 * *$ & $0,61 * *$ & $-0,17^{\mathrm{ns}}$ & $0,69 * *$ & $-0,55 * *$ & $0,46^{*}$ & $0,70 * *$ \\
\hline $\mathrm{CP}$ & $0,13^{\mathrm{ns}}$ & $0,25^{\mathrm{ns}}$ & - & $0,38^{*}$ & $0,36^{*}$ & $0,09^{\mathrm{ns}}$ & $0,33^{\text {ns }}$ & $0,26^{\mathrm{ns}}$ & $-0,30^{\mathrm{ns}}$ & $0,02^{\mathrm{ns}}$ & $0,66^{* *}$ \\
\hline DP & $0,74 * *$ & $0,40 *$ & $0,42 *$ & - & $0,69 * *$ & $0,31^{\mathrm{ns}}$ & $0,26^{\mathrm{ns}}$ & $0,58 * *$ & $-0,24^{\mathrm{ns}}$ & $-0,02^{\mathrm{ns}}$ & $0,67 * *$ \\
\hline $\mathrm{AF}$ & $0,77 * *$ & $0,82 * *$ & $0,37^{*}$ & $0,71 * *$ & - & $0,65^{* *}$ & $-0,08^{\mathrm{ns}}$ & $0,80 * *$ & $-0,60 * *$ & $0,34^{\mathrm{ns}}$ & $0,81 * *$ \\
\hline AP & $0,50 * *$ & $0,71 * *$ & $0,10^{\mathrm{ns}}$ & $0,34^{\mathrm{ns}}$ & $0,73 * *$ & - & $-0,50 * *$ & $0,60 * *$ & $-0,60 * *$ & $0,53 * *$ & $0,44 *$ \\
\hline $\mathrm{DC}$ & $0,10^{\mathrm{ns}}$ & $-0,24^{\mathrm{ns}}$ & $0,33^{\mathrm{ns}}$ & $0,24^{\mathrm{ns}}$ & $-0,15^{\mathrm{ns}}$ & $0,55 * *$ & - & $0,00^{\mathrm{ns}}$ & $0,15^{\mathrm{ns}}$ & $-0,45^{*}$ & $0,09^{\mathrm{ns}}$ \\
\hline NFC & $0,82 * *$ & $0,77 * *$ & $0,25^{\mathrm{ns}}$ & $0,63 * *$ & $0,89 * *$ & $0,72 * *$ & $-0,07^{\mathrm{ns}}$ & - & $-0,40 *$ & $0,31^{\mathrm{ns}}$ & $0,54 * *$ \\
\hline NFT & $-0,65^{* *} *$ & $-0,64 * *$ & $-0,32^{\mathrm{ns}}$ & $-0,27^{\mathrm{ns}}$ & $-0,69 * *$ & $-0,68 * *$ & $0,15^{\text {ns }}$ & $-0,46^{*}$ & - & $-0,54 * *$ & $-0,64 * *$ \\
\hline $\mathrm{NB}$ & $0,21^{\mathrm{ns}}$ & $0,59 * *$ & $0,02^{\mathrm{ns}}$ & $0,01^{\mathrm{ns}}$ & $0,45^{*}$ & $0,61 * *$ & $-0,53 * *$ & $0,42 *$ & $-0,63 * *$ & - & $0,23^{\mathrm{ns}}$ \\
\hline MFS & $0,61 * *$ & $0,81 * *$ & $0,72^{* *}$ & $0,73 * *$ & $0,91 * *$ & $0,48^{*}$ & $0,05^{\text {ns }}$ & $0,61 * *$ & $-0,70 * *$ & $0,26^{\mathrm{ns}}$ & - \\
\hline
\end{tabular}

$\mathrm{CF}$, comprimento de folha; LF, largura de folha; CP, comprimento de pecíolo; DP, diâmetro do centro do pecíolo; AF, área foliar; AP, altura de planta; DC, diâmetro de caule; NFC, número de folhas comerciais; NTF, número total de folhas; e NB, número de brotações; MSF, massa de matéria seca de folhas. **,

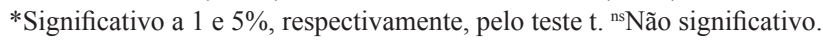


Quanto ao diâmetro do caule, verificou-se correlação negativa significativa com a altura de plantas e o número de brotações, e correlações não significativas com as outras características. O diâmetro do caule tem considerável importância no melhoramento, pois, segundo Novo et al. (2010b), ele está relacionado à perda de plantas pela ação do vento. No entanto, Chakwizira (2007) relatam que o diâmetro do caule é significativamente influenciado pelas condições climáticas da região de cultivo e, segundo Trani (2008), pelos tratos culturais, especialmente a adubação nitrogenada.

Para o número de folhas comerciais, característica mais importante para o melhoramento, verificou-se correlação negativa significativa com o número total de folhas (Tabela 2). Comprimento de pecíolo, diâmetro de caule e número de brotações não se correlacionaram com o número de folhas comerciais, mas os demais caracteres apresentaram valores de correlação positivos e significativos com essa característica.

Os genótipos UFVJM-10 e UFLA-11, seguidos de UFLA-1 e UFLA-3 destacaram-se quanto à dimensão do limbo foliar (Tabela 3). Em geral, os genótipos não comerciais se sobressaíram na comparação com os comerciais, no que se refere a essa característica. Comportamento similar foi observado quanto ao comprimento e diâmetro de pecíolo, em que os genótipos comerciais apresentaram menores dimensões. Quanto à dimensão de pecíolo, o genótipo UFLA-11, seguido de UFVJM-24, UFLA-6 e UFLA-12, destacouse, com maiores valores de comprimento e diâmetro. Para a comercialização de folhas de couve, genótipos com pecíolos muito grandes são indesejáveis, pois eles são normalmente descartados e dificultam o transporte.

Tabela 3. Média de comprimento de folha, largura de folha, comprimento de pecíolo, diâmetro do centro do pecíolo, área foliar e massa de matéria seca de folhas em genótipos de couve ${ }^{(1)}$.

\begin{tabular}{|c|c|c|c|c|c|c|}
\hline Genótipo & $\begin{array}{l}\text { Comprimento de } \\
\text { folha }(\mathrm{cm})\end{array}$ & $\begin{array}{l}\text { Largura de } \\
\text { folha }(\mathrm{cm})\end{array}$ & $\begin{array}{l}\text { Área foliar } \\
\left(\mathrm{cm}^{2}\right)\end{array}$ & $\begin{array}{l}\text { Comprimento de } \\
\text { pecíolo }(\mathrm{cm})\end{array}$ & $\begin{array}{c}\text { Diâmetro de } \\
\text { pecíolo }(\mathrm{mm})\end{array}$ & $\begin{array}{l}\text { Massa de matéria } \\
\text { seca de folhas }(\mathrm{g})\end{array}$ \\
\hline UFVJM-2 & $17,55 b$ & $14,08 \mathrm{a}$ & $206,56 \mathrm{c}$ & $6,03 \mathrm{e}$ & $6,36 \mathrm{c}$ & $3,10 \mathrm{~b}$ \\
\hline UFVJM-3 & $19,25 b$ & $11,78 \mathrm{~b}$ & $193,35 c$ & $9,99 b$ & $6,69 \mathrm{c}$ & $3,12 b$ \\
\hline UFVJM-4 & $17,53 b$ & $16,00 \mathrm{a}$ & $225,64 b$ & $6,66 \mathrm{~d}$ & $6,99 b$ & $3,07 b$ \\
\hline UFVJM-5 & $14,58 \mathrm{c}$ & $13,54 a$ & $168,06 \mathrm{c}$ & $6,07 \mathrm{e}$ & $5,31 \mathrm{c}$ & $2,61 \mathrm{c}$ \\
\hline UFVJM-7 & $16,03 b$ & $12,50 \mathrm{~b}$ & $167,85 \mathrm{c}$ & $6,58 \mathrm{~d}$ & $7,08 \mathrm{~b}$ & $2,30 \mathrm{c}$ \\
\hline UFVJM-8 & $16,69 b$ & $15,71 \mathrm{a}$ & $201,34 \mathrm{c}$ & $6,12 \mathrm{e}$ & $7,11 \mathrm{~b}$ & $2,34 \mathrm{c}$ \\
\hline UFVJM-9 & $16,26 b$ & $14,60 \mathrm{a}$ & $181,38 \mathrm{c}$ & $6,02 \mathrm{e}$ & $6,09 \mathrm{c}$ & $2,96 \mathrm{~b}$ \\
\hline UFVJM-10 & $23,58 \mathrm{a}$ & $14,23 \mathrm{a}$ & $276,27 \mathrm{a}$ & $5,47 \mathrm{e}$ & $7,93 b$ & $3,06 \mathrm{~b}$ \\
\hline UFVJM-13 & $15,78 b$ & $13,98 \mathrm{a}$ & $187,89 \mathrm{c}$ & $6,53 \mathrm{~d}$ & $6,38 \mathrm{c}$ & $2,39 \mathrm{c}$ \\
\hline UFVJM-19 & $17,53 b$ & $15,63 \mathrm{a}$ & $222,78 b$ & $6,65 d$ & $6,78 \mathrm{c}$ & $2,61 \mathrm{c}$ \\
\hline UFVJM-21 & $17,46 b$ & $13,91 \mathrm{a}$ & $194,20 \mathrm{c}$ & $5,20 \mathrm{e}$ & $6,81 \mathrm{c}$ & $2,91 \mathrm{~b}$ \\
\hline UFVJM-22 & $17,53 b$ & $15,23 \mathrm{a}$ & $210,29 b$ & $6,50 \mathrm{~d}$ & $6,65 c$ & $3,19 b$ \\
\hline UFVJM-24 & $13,48 \mathrm{c}$ & $13,50 \mathrm{a}$ & $189,22 \mathrm{c}$ & $11,60 \mathrm{a}$ & $7,56 \mathrm{~b}$ & $3,65 \mathrm{~b}$ \\
\hline UFVJM-26 & $21,51 \mathrm{a}$ & $14,35 \mathrm{a}$ & $190,84 \mathrm{c}$ & $5,83 \mathrm{e}$ & $6,41 \mathrm{c}$ & $2,41 \mathrm{c}$ \\
\hline UFVJM-27 & $16,54 b$ & $13,76 \mathrm{a}$ & $188,85 \mathrm{c}$ & $5,97 \mathrm{e}$ & $5,67 \mathrm{c}$ & $2,24 \mathrm{c}$ \\
\hline UFVJM-30 & $18,21 b$ & $15,83 \mathrm{a}$ & $237,78 b$ & $6,98 \mathrm{~d}$ & $6,88 b$ & $2,83 \mathrm{~b}$ \\
\hline UFVJM-32 & $17,93 b$ & $15,43 a$ & $229,55 b$ & $6,60 \mathrm{~d}$ & $7,42 b$ & $3,29 b$ \\
\hline UFVJM-34 & $16,78 b$ & $13,90 \mathrm{a}$ & $174,61 \mathrm{c}$ & $5,25 \mathrm{e}$ & $6,47 \mathrm{c}$ & $2,75 b$ \\
\hline UFVJM-36 & $18,28 \mathrm{~b}$ & $15,15 \mathrm{a}$ & $234,93 b$ & $6,43 \mathrm{~d}$ & $7,43 b$ & $3,39 b$ \\
\hline UFLA-1 & $19,97 \mathrm{a}$ & $15,06 \mathrm{a}$ & $244,13 b$ & $5,75 \mathrm{e}$ & $7,51 b$ & $3,30 \mathrm{~b}$ \\
\hline UFLA-3 & $22,81 \mathrm{a}$ & $14,07 \mathrm{a}$ & $243,00 \mathrm{~b}$ & $5,68 \mathrm{e}$ & $10,31 \mathrm{a}$ & $3,17 \mathrm{~b}$ \\
\hline UFLA-5 & $14,48 \mathrm{c}$ & $14,93 \mathrm{a}$ & $195,98 \mathrm{c}$ & $7,93 \mathrm{~d}$ & $6,39 c$ & $2,93 b$ \\
\hline UFLA-6 & $18,61 b$ & $12,85 b$ & $224,62 b$ & $10,44 b$ & $7,50 \mathrm{~b}$ & $3,24 b$ \\
\hline UFLA-8 & $18,14 b$ & $16,21 \mathrm{a}$ & $248,68 b$ & $8,73 \mathrm{c}$ & $7,26 \mathrm{~b}$ & $3,34 \mathrm{~b}$ \\
\hline UFLA-10 & $16,10 \mathrm{~b}$ & $15,21 \mathrm{a}$ & $219,05 b$ & $8,33 \mathrm{c}$ & $6,67 \mathrm{c}$ & $2,84 b$ \\
\hline UFLA-11 & $23,83 \mathrm{a}$ & $18,48 \mathrm{a}$ & $317,25 \mathrm{a}$ & $12,48 \mathrm{a}$ & $9,68 \mathrm{a}$ & $5,61 \mathrm{a}$ \\
\hline UFLA-12 & $17,38 b$ & $14,75 \mathrm{a}$ & $199,73 \mathrm{c}$ & $9,38 b$ & $7,81 \mathrm{~b}$ & $3,29 b$ \\
\hline COM-1 & $13,62 \mathrm{c}$ & $10,95 b$ & $124,09 \mathrm{~d}$ & $4,78 \mathrm{e}$ & $6,21 \mathrm{c}$ & $1,61 d$ \\
\hline COM-2 & $17,22 b$ & $10,57 b$ & $111,22 d$ & $7,59 \mathrm{~d}$ & $6,22 \mathrm{c}$ & $1,80 \mathrm{~d}$ \\
\hline COM-3 & $13,26 \mathrm{c}$ & $11,33 b$ & $119,03 \mathrm{~d}$ & $4,83 \mathrm{e}$ & $6,14 \mathrm{c}$ & $1,72 \mathrm{~d}$ \\
\hline
\end{tabular}

${ }^{(1)}$ Médias seguidas de letras iguais não diferem pelo teste de Scott Knott, a 5\% de probabilidade. 
Quanto à massa de matéria seca de folhas, o genótipo UFLA-11 apresentou maior valor, e os genótipos comerciais (COM-1, COM-2 e COM-3) tiveram médias inferiores aos demais.

Quanto à altura da planta, sobressaíram-se os acessos UFVJM-27 e UFLA-3 com as maiores médias. Antigamente os agricultores preferiam plantas mais altas, em razão da facilidade de colheita das folhas (Nieuwhof, 1969). Contudo, atualmente, a preferência por genótipos de menor porte vem crescendo, e as cultivares comerciais destacaram-se pelo baixo porte (Tabela 4). Quanto ao diâmetro do caule, a cultivar couve-manteiga Baby e o genótipo UFLA-11 apresentaram maiores valores, o que é desejável, por diminuir a necessidade de tutoramento. Os genótipos que apresentaram maior número de folhas totais foram os genótipos comerciais 900 e Geórgia; no entanto, eles figuraram entre os genótipos com menor número de folhas comerciais. Para esta característica, destacaramse os acessos UFVJM-4, UFVJM-8, UFVJM-10, UFVJM-13, UFVJM-19, UFVJM-22, UFVJM-26, UFVJM-30, UFLA-1, UFLA-3, UFLA-5, UFLA-8, UFLA-10, UFLA-11 e UFLA-12.

O genótipos UFLA-6 e as cultivares comerciais apresentaram as menores médias de brotações (Tabela 4). A seleção de genótipos de couve com menor número de brotações pode ser usado pelas empresas produtoras de sementes, para reduzir o potencial de propagação vegetativa da planta, o que garantiria a venda contínua de sementes. Outra vantagem do menor número de brotações consiste na redução dos tratos culturais referente à desbrota, o que reduz os custos com mão de obra para a produção comercial de folhas.

Tabela 4. Altura de planta, diâmetro de caule, número total de folhas, número de folhas comerciais e número de brotações em genótipos de couve ${ }^{(1)}$.

\begin{tabular}{|c|c|c|c|c|c|}
\hline Genótipo & Altura de planta $(\mathrm{cm})$ & Diâmetro do caule $(\mathrm{cm})$ & № total de folhas & № de folhas comerciais & № de brotações \\
\hline UFVJM-2 & $59,45 \mathrm{c}$ & $10,94 d$ & $9,42 \mathrm{c}$ & $1,42 b$ & $6,75 \mathrm{a}$ \\
\hline UFVJM-3 & $68,65 b$ & $12,04 \mathrm{c}$ & $9,75 \mathrm{c}$ & $1,75 b$ & $4,92 \mathrm{a}$ \\
\hline UFVJM-4 & $66,35 b$ & $12,80 \mathrm{c}$ & $9,33 \mathrm{c}$ & $2,17 \mathrm{a}$ & $4,92 \mathrm{a}$ \\
\hline UFVJM-5 & $59,50 \mathrm{c}$ & $8,70 \mathrm{~d}$ & $9,50 \mathrm{c}$ & $0,92 b$ & $6,17 \mathrm{a}$ \\
\hline UFVJM-7 & $58,10 \mathrm{c}$ & $11,90 \mathrm{c}$ & $14,08 b$ & $2,17 b$ & $7,42 \mathrm{a}$ \\
\hline UFVJM-8 & $67,75 b$ & $12,57 \mathrm{c}$ & $11,58 b$ & $2,83 \mathrm{a}$ & $4,83 \mathrm{a}$ \\
\hline UFVJM-9 & $57,65 \mathrm{c}$ & $11,19 d$ & $9,25 \mathrm{c}$ & $1,75 b$ & $6,42 \mathrm{a}$ \\
\hline UFVJM-10 & $64,65 \mathrm{c}$ & $12,55 \mathrm{c}$ & $9,67 \mathrm{c}$ & $4,42 \mathrm{a}$ & $6,25 \mathrm{a}$ \\
\hline UFVJM-13 & $63,75 \mathrm{c}$ & $9,15 \mathrm{~d}$ & $13,50 \mathrm{~b}$ & $2,67 \mathrm{a}$ & $8,83 a$ \\
\hline UFVJM-19 & $67,05 b$ & $10,92 d$ & $11,67 b$ & $2,75 \mathrm{a}$ & $7,33 \mathrm{a}$ \\
\hline UFVJM-21 & $69,30 \mathrm{~b}$ & $11,89 \mathrm{c}$ & $10,42 \mathrm{c}$ & $1,75 b$ & $4,83 \mathrm{a}$ \\
\hline UFVJM-22 & $70,10 \mathrm{~b}$ & $10,68 \mathrm{~d}$ & $9,25 \mathrm{c}$ & $2,50 \mathrm{a}$ & $4,08 \mathrm{a}$ \\
\hline UFVJM-24 & $54,20 \mathrm{c}$ & $14,92 \mathrm{c}$ & $12,00 \mathrm{~b}$ & $1,25 b$ & $7,00 \mathrm{a}$ \\
\hline UFVJM-26 & $69,25 b$ & $11,00 \mathrm{~d}$ & $10,08 \mathrm{c}$ & $2,67 \mathrm{a}$ & $6,67 a$ \\
\hline UFVJM-27 & $83,35 a$ & $11,28 \mathrm{~d}$ & $10,50 \mathrm{c}$ & $2,00 \mathrm{~b}$ & $6,33 a$ \\
\hline UFVJM-30 & $69,90 \mathrm{~b}$ & $12,06 \mathrm{c}$ & $13,00 \mathrm{~b}$ & $2,50 \mathrm{a}$ & $6,92 \mathrm{a}$ \\
\hline UFVJM-32 & $64,05 \mathrm{c}$ & $11,92 \mathrm{c}$ & $10,42 \mathrm{c}$ & $1,50 \mathrm{~b}$ & $5,83 \mathrm{a}$ \\
\hline UFVJM-34 & $73,25 b$ & $10,25 d$ & $9,75 \mathrm{c}$ & $1,17 b$ & $6,00 \mathrm{a}$ \\
\hline UFVJM-36 & $68,85 b$ & $12,84 \mathrm{c}$ & $9,92 \mathrm{c}$ & $1,83 b$ & $5,58 \mathrm{a}$ \\
\hline UFLA-1 & $65,20 \mathrm{c}$ & $12,43 \mathrm{c}$ & $10,08 \mathrm{c}$ & $2,83 \mathrm{a}$ & $4,92 \mathrm{a}$ \\
\hline UFLA-3 & $79,26 \mathrm{a}$ & $13,12 \mathrm{c}$ & $11,50 \mathrm{~b}$ & $3,33 \mathrm{a}$ & $4,25 \mathrm{a}$ \\
\hline UFLA-5 & $71,60 \mathrm{~b}$ & $12,70 \mathrm{c}$ & $13,42 b$ & $3,67 \mathrm{a}$ & $5,08 \mathrm{a}$ \\
\hline UFLA-6 & $68,70 \mathrm{~b}$ & $11,54 d$ & $10,08 \mathrm{c}$ & $2,08 b$ & $1,67 b$ \\
\hline UFLA-8 & $62,80 \mathrm{c}$ & $14,19 \mathrm{c}$ & $10,33 \mathrm{c}$ & $3,33 \mathrm{a}$ & $5,42 \mathrm{a}$ \\
\hline UFLA-10 & $67,25 b$ & $12,56 \mathrm{c}$ & $11,00 \mathrm{c}$ & $2,42 \mathrm{a}$ & $4,83 a$ \\
\hline UFLA-11 & $69,30 \mathrm{~b}$ & $17,07 \mathrm{~b}$ & $8,33 \mathrm{c}$ & $4,42 \mathrm{a}$ & $4,33 \mathrm{a}$ \\
\hline UFLA-12 & $63,90 \mathrm{c}$ & $10,24 d$ & $9,58 \mathrm{c}$ & $2,17 \mathrm{a}$ & $6,42 \mathrm{a}$ \\
\hline COM-1 & $35,40 \mathrm{~d}$ & $13,49 \mathrm{c}$ & $20,58 \mathrm{a}$ & $0,50 \mathrm{~b}$ & $0,67 \mathrm{c}$ \\
\hline COM-2 & $31,20 \mathrm{~d}$ & $22,39 a$ & $11,83 \mathrm{~b}$ & $1,08 \mathrm{~b}$ & $3,08 \mathrm{~b}$ \\
\hline COM-3 & $36,40 \mathrm{~d}$ & $13,86 \mathrm{c}$ & $20,50 \mathrm{a}$ & $0,67 \mathrm{~b}$ & $0,75 \mathrm{c}$ \\
\hline
\end{tabular}

${ }^{(1)}$ Médias seguidas de letras iguais não diferem pelo teste de Scott Knott, a 5\% de probabilidade. 
A diferença dos genótipos comerciais para os acessos, observada na maioria das características avaliadas, se justifica pelo interesse dos programas de melhoramento em selecionar genótipos com menor altura, menor número de brotações e maior número de folhas. No entanto, esta última característica está negativamente correlacionada com a dimensão da folha e, consequentemente, com o número de folhas comerciais. Além deste inconveniente, os genótipos comerciais propagados por sementes apresentam plantas de porte compacto e normalmente são pouco atrativas para o consumidor (Novo et al., 2010b), por apresentarem folhas com coloração mais escura, nervuras proeminentes e de cor clara, características que o consumidor associa ao maior tempo de cocção.

\section{Conclusões}

1. Há variabilidade genética entre os genótipos, com predominância dos efeitos genéticos sobre os ambientais, o que indica ser possível obter ganhos genéticos com o melhoramento.

2. As características comprimento e largura de folhas, diâmetro de pecíolo, área foliar, altura de planta, número total de folhas, número de brotações e massa de matéria seca de folhas correlacionam-se significativamente com a produção de folhas comerciais e são, portanto, importantes para a seleção indireta, no melhoramento genético da couve.

3. Os genótipos comerciais apresentam menores áreas foliares, massa de folhas secas e altura de planta, e figuram entre os genótipos com menor comprimento e largura de folha, comprimento de pecíolo, número de folhas comerciais e número de brotações.

\section{Agradecimentos}

À Fundação de Amparo à Pesquisa do Estado de Minas Gerais (Fapemig), ao Conselho Nacional de Desenvolvimento Científico e Tecnológico $(\mathrm{CNPq})$ e à Coordenação de Aperfeiçoamento de Pessoal de Nível Superior (Capes), por concessão de bolsas e apoio financeiro; à Universidade Federal de Lavras (UFLA) pela disponibilização dos genótipos código UFLA.

\section{Referências}

BALKAYA, A.; YANMAZ, R. Promising kale (Brassica oleracea var. acephala) populations from Black Sea region, Turkey. New Zealand Journal of Crop and Horticultural Science, v.33, p.1-7, 2005.
BELETE, Y.S.; KEBEDE, S.A.; GEMELAL, A.W. Multivariate analysis of genetic divergence among Ethiopian mustard (Brassica carinata A. Brun) genotypes in relation to seed oil quality traits. International Journal of Agricultural Research, v.6, p.494-503, 2011.

CARTEA, M.E.; PICOAGA, A.; SOENGAS, P.; ORDÁS, A. Morphological characterization of kale populations from Northwestern Spain. Euphytica, v.129, p.25-32, 2002.

CHAKWIZIRA, E. Growth and development of 'Pasja' and kale crops with two methods and four rates of phosphorus (P) application. 2008. 127p. Tese (Mestrado) - Lincoln University, Lincoln.

CHRISTENSEN, S.; BOTHMER, R.V.; POULSEN, G.; MAGGIONI, L.; PHILLIP, M.; ANDERSEN, B.A.; JØRGENSEN, R.B. AFLP analysis of genetic diversity in leafy kale (Brassica oleracea L. convar. acephala (DC.) Alef.) landraces, cultivars and wild populations in Europe. Genetic Resources and Crop Evolution, v.58, p.657-666, 2011.

CRUZ, C.D.; REGAZZI, A.J.; CARNEIRO, P.C.S. Modelos biométricos aplicados ao melhoramento genético. 3.ed. Viçosa: UFV, 2004. 480p.

FILGUEIRA, F.A.R. Novo manual de olericultura: agrotecnologia moderna na produção e comercialização de hortaliças. 2.ed. Viçosa: UFV, 2008. 421p.

FREITAS, M.L.M.; SEBBENN, A.M.; ZANATTO, A.C.S.; MORAES, E. Pomar de sementes por mudas a partir da seleção dentro em teste de progênies de Myracrodruon urundeuva Fr. All. Revista do Instituto Florestal, v.19, p.65-72, 2007.

KHAN, S.H.; AHMED, N.; JABEEN, N.; MUSHTAK, F.; HSSAIN, $\mathrm{K}$. Genetic divergence in kale (Brassica oleracea var. acephala L.) S.H. Asian Journal of Horticulture, v.4, p.134-137, 2009.

KHATUN, H.; RASUL, M.G.; SAIKAT, M.M.H.; HAQUE, M.M. Genetic diversity in Brassica rapa L. Bangladesh Journal of Plant Breeding and Genetics, v.23, p.31-37, 2010.

KOUTITA, O.; TERTIVANIDIS, K.; KOUTSOS, T.; KOUTSIKA-SOTIRIOU, M.; SKARACIS, G. Genetic changes between $\mathrm{C}_{0}, \mathrm{C}_{3}, \mathrm{C}_{5}$ cycles of selection and $\mathrm{S}_{4}$ lines of two cabbage (Brassica oleracea L. var. capitata L.) open pollinated populations based on RAPD markers. Biotechnology, v.7, p.475-480, 2008.

MORENO, D.A.; CARVAJAL, M.; LOPEZ-BERENGUER, C.; GARCIA-VIGUERA, C. Chemical and biological characterization of nutraceutical compounds of broccoli. Journal of Pharmaceutical and Biomedical Analysis, v.41, p.1508-1522, 2006.

NATH, U.K.; NAZ, S.; RAHMAN, M.M. Genetic divergence of Brassica campestris, Brassica juncea parents and their hybrids. Pakistan Journal of Biological Sciences, v.6, p.936-938, 2003.

NIEUWHOF, M. Cole crops. London: World Crops Books, 1969. $95 \mathrm{p}$.

NOVO, M. do C. de S.S.; PRELA-PANTANO, A.; DEUBER, R.; TORRES, R.B.; TRANI, P.E.; BRON, I.U. Morfologia de folhas de couve do Banco de Germoplasma do Instituto Agronômico. Campinas: Instituto Agronômico de Campinas, 2010b. 27p. 
NOVO, M. do C. de S.S.; PRELA-PANTANO, A.; TRANI, P.E.; BLAT, S.F. Desenvolvimento e produção de genótipos de couve-manteiga. Horticultura Brasileira, v.28, p.321-325, 2010a.

OKUMUS, A.; BALKAYA, A. Estimation of genetic diversity among Turkish kale populations (Brassica oleracea var. acephala L.) using RAPD markers. Russian Journal of Genetics, v.43, p.411-415, 2007.

SATO, A.S.; SEBBENN, A.M.; MORAES, E.; ZANATTO, A.C.S.; FREITAS, M.L.M. Seleção dentro de progênies de Eucalyptus resinifera aos 21 anos de idade em Luiz Antônio - SP. Revista do Instituto Florestal, v.19, p.93-100, 2007.

SAWAZAKI, H.E.; NAGAI, H.; SODEK, L. Caracterização da variabilidade genética em couve-manteiga utilizando isoenzimas e RAPD. Bragantia, v.56, p.6-19, 1997.
SJÖDIN, P.; HEDMAN, H.; OSTERBERG, M.K.; GUSTAFSSON, S.; LAGERCRANTZ U.; LASCOUX M. Polymorphism and divergence at three duplicate genes in Brassica nigra. Journal of Molecular Evolution, v.66, p.581-590, 2008.

TRANI, P.E. Avaliação agronômica, organoléptica e caracterização botânica da coleção de germoplasma de couve de folhas do IAC. São Paulo: Secretaria de Agricultura e Abastecimento, 2008. 3p.

VAISHNAVA, A.; SACHAN, J.N.; TEWARI, S.K. Genetic divergence for important quantitative traits in Indian mustard \{Brassica juncea (L.) Czern and Coss\}. Agricultural Science Digest, v.26, p.269-272, 2006.

YOUSUF, M.; AJMAL, S.U.; MUNIR, M.; GHAFOOR, A. Genetic diversity analysis for agro-morphological and seed quality traits in rapeseed (Brassica campestris L.). Pakistan Journal of Botany, v.43, p.1195-1203, 2011.

$\overline{\text { Recebido em } 6 \text { de maio de } 2012 \text { e aprovado em } 15 \text { de outubro de } 2012}$ 